\title{
Broad Spectrum Polyphenol Supplementation from Tart Cherry Extract on Markers of Recovery from Intense Resistance Exercise
}

\author{
D. R. Hooper ${ }^{1,2^{*}}$ (D) T. Orange ${ }^{1}$, M. T. Gruber ${ }^{3}$, A. A. Darakjian ${ }^{3}$, K. L. Conway ${ }^{3}$ and H. A. Hausenblas ${ }^{1,2}$
}

\begin{abstract}
Background: Tart cherry supplementation has been shown to enhance recovery from strenuous exercise due to its antioxidant properties. The majority of these studies used tart cherry juice, with a significant calorie content. The primary purpose of this study was to assess whether powdered tart cherry extract with minimal calorie content reduces oxidative stress and enhances recovery following intense resistance exercise.

Methods: Thirteen men (mean age: $26.2 \pm 5.3$ years; height: $184.3 \pm 8.2 \mathrm{~cm}$; weight: $92.9 \pm 15.6 \mathrm{~kg}$ ) performed a demanding resistance exercise protocol consisting of 6 sets of 10 repetitions of barbell back squat with $80 \% 1 \mathrm{RM}$. The protocol was performed once following 7 days of $500 \mathrm{mg}$ of tart cherry extract and once following placebo. Serum protein carbonyl (PC) content, creatine kinase activity (CK) and creatine kinase myocardial band content (CK-MB) were used to assess oxidative stress, skeletal and cardiac muscle damage respectively. Muscle soreness was assessed by visual analog scale. Physical performance was measured by countermovement jump power and handgrip dynamometer strength.

Results: There was a significant increase in $\mathrm{PC}$ in the placebo $(\mathrm{PL})$ condition when compared to the Tart Cherry (TC)

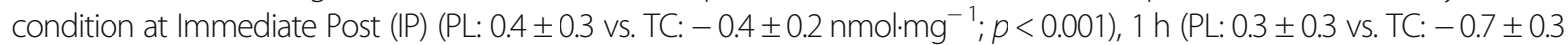

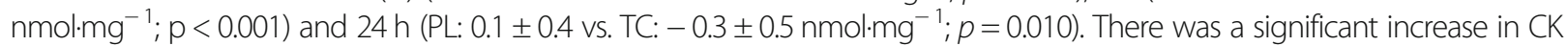
activity in PL when compared to the TC at IP (PL: $491.1 \pm 280$ vs. TC: $\left.296.3 \pm 178 \mathrm{U} \cdot \mathrm{L}^{-1} ; p=0.008\right)$ and $3 \mathrm{~h}$ (PL: $-87 \pm 123$ vs. $\left.\mathrm{TC}: 43.1 \pm 105.3 \mathrm{U} \cdot \mathrm{L}^{-1} ; p=0.006\right)$. There was a significant $(p=0.003)$ increase in CKMB concentration in PL when compared to the TC (PL: $21.6 \pm 12.4$ vs. TC: $-0.3 \pm 11.8 \mathrm{ng} \cdot \mathrm{ml}^{-1} ; p=0.006$ ) at $1 \mathrm{~h}$ post. There was a significant increase in handgrip strength in TC when compared to PL (PL: $-2 \pm 5.1$ vs. TC: $1.7 \pm 3 \mathrm{~kg} ; p=0.017$ ) at $24 \mathrm{~h}$ post.

Conclusions: This study demonstrated that tart cherry extract reduced oxidative stress and markers of muscle and cardiac damage following intense resistance exercise. This occurred along with a prevention of the decrease in handgrip strength seen following the intense exercise protocol, indicating a potential reduction in central fatigue. These benefits were seen with minimal energy intake.
\end{abstract}

Keywords: Tart cherry, antioxidants, polyphenols, recovery, muscle damage, cardiac damage

\footnotetext{
* Correspondence: dhooper4@ju.edu

${ }^{1}$ Center for Health and Human Performance, Jacksonville University, Jacksonville, USA

${ }^{2}$ Department of Kinesiology, Brooks Rehabilitation College of Healthcare

Sciences, Health Sciences Complex, Jacksonville University, 2800 University Boulevard North, Jacksonville, FL 32211, USA

Full list of author information is available at the end of the article
}

C C The Author(s). 2021 Open Access This article is licensed under a Creative Commons Attribution 4.0 International License, which permits use, sharing, adaptation, distribution and reproduction in any medium or format, as long as you give appropriate credit to the original author(s) and the source, provide a link to the Creative Commons licence, and indicate if changes were made. The images or other third party material in this article are included in the article's Creative Commons licence, unless indicated otherwise in a credit line to the material. If material is not included in the article's Creative Commons licence and your intended use is not permitted by statutory regulation or exceeds the permitted use, you will need to obtain permission directly from the copyright holder. To view a copy of this licence, visit http://creativecommons.org/licenses/by/4.0/. The Creative Commons Public Domain Dedication waiver (http://creativecommons.org/publicdomain/zero/1.0/) applies to the data made available in this article, unless otherwise stated in a credit line to the data. 


\section{Background}

Resistance exercise is a powerful stimulus to induce gains in strength and hypertrophy [1]. Although such gains can lead to enhanced athletic performance and may reduce the demands of activities of daily living, paradoxically resistance exercise increases markers of oxidative stress [2,3], skeletal [4] and cardiac [5] muscle damage, and transiently reduces physical performance [6]. These responses to exercise are not necessarily negative, but rather are a necessary part of the adaptation process, providing the level of stress does not exceed the ability of the body to recover.

However, if the stress caused by exercise is too great and the body is unable to recover adequately, long term reductions in performance level can occur, such as in non-functional overreaching, or overtraining [7], or in much more severe cases, can be life threatening in the form of rhabdomyolysis [8]. Supplementation with plant compounds has been suggested to be able to reduce inflammation, oxidative stress and subsequent skeletal and cardiac muscle damage associated with intense exercise, thereby enhancing recovery and helping to stimulate optimal adaptation.

One such group of plant compounds are polyphenols, which are naturally high in fruits and vegetables. Although polyphenols themselves are radical scavengers, they exist in such low concentrations in human blood that their ability to reduce markers of oxidative stress are more likely due to their capacity to enhance endogenous anti-inflammatory and antioxidant mechanisms through the nuclear factor erythroid 2-related factor 2 (Nrf2) pathway than by directly scavenging themselves [9].

One source of polyphenols that has received considerable attention in this area is tart cherries [3, 6, 10-16]. Following various forms of intense physical activity, such as resistance exercise $[3,6,13]$, long distance running $[14,16]$, cycling $[11,12]$, repeated sprints [10], or sport [15], consumption of tart cherries reduces markers of oxidative stress $[3,11,16]$, muscle soreness $[10,13,14]$ and attenuates reductions in physical performance [3, $10,12,13,16]$. These results are not unequivocal, however, likely due to differences in dosing and damage protocol [9]. Complicating the interpretation further, the enhanced recovery is not always seen as some studies failed to show either an increase in muscle damage [11], or a reduction in performance [15] from the chosen protocol, giving the supplement no damage/performance measure to recover from.

While there are many ways to assess oxidative stress, one particular method that has been used to demonstrate the effectiveness of a polyphenol supplement to reduce its effects following exercise has been by a decrease in serum protein carbonyls (PC), a marker of oxidative damage [2]. For example, Bowtell et al. [3] saw a significant reduction in PC following 10 sets of 10 knee extensions at $80 \% 1 \mathrm{RM}$ following a Montmorency cherry supplementation when compared to an isoenergetic fruit concentrate. Also, Chang et al. [17] demonstrated a reduction in PC following $1 \mathrm{~h}$ of treadmill running with the use of a polyphenol supplementation in the form of purple sweet potato when compared to control.

One variable not measured in prior polyphenol research, but has been assessed in other antioxidant literature, is creatine kinase myocardial band (CK-MB), a marker typically associated with cardiac damage [5] that has recently been proposed as a marker specifically for type I muscle fiber damage [18]. An increase in CK-MB is a typical response to exercise and is associated with the structural disruption of muscle fibers and the leaking of the contents on these fibers into the plasma [5]. Strenuous exercise, such as long distance running [18], professional soccer [19] and also resistance exercise [5] significantly increases CK-MB. These increases have been attenuated by other antioxidant supplements, such as Vitamin E under hypoxic conditions [20] and the drug Allopurinol [19], and thus antioxidants could potentially enhance recovery from strenuous exercise in the context of CK-MB.

While it stands to reason that aerobic activity, when compared with anaerobic activity, would be the more likely beneficiary of an antioxidant supplementation due to its reliance on oxidative metabolism, oxidative stress has been demonstrated following resistance training and there are alternative ways that oxidative stress can impact recovery. For example, many studies have assessed the impact of polyphenol supplementation on skeletal muscle damage in the form of changes in serum creatine kinase $(\mathrm{CK})$ and/or muscle soreness. The prevailing rationale for such analyses is that while mechanical stress is a major contributor to skeletal muscle damage, an increase in reactive oxygen species could cause oxidative modifications of skeletal muscle proteins. Thus, an antioxidant could potentially reduce biochemical damage [9]. This rationale has received equivocal support, with some studies showing a benefit of polyphenol supplementation $[6,14,21-23]$ and others not $[3,11,12,16$, 24-26]. In terms of the studies that utilized resistance exercise, outside of Levers et al. [6], all utilized unilateral strength exercise or parallel group design, which are susceptible to high inter-individual differences and could explain the lack of agreement in the literature [9].

While changes in oxidative stress and/or markers of muscle damage would be promising to demonstrate whether polyphenol supplementation is efficacious, it would be important to demonstrate a measureable improvement in physical performance level resulting from that reduced oxidative stress. While the majority of 
research has demonstrated some form of enhanced performance level during the recovery process as a result of polyphenol supplementation, such as maximal voluntary contraction [3, 12, 24, 27], isokinetic performance [21, 25] or time trial performance [10], some studies have failed to show performance benefits $[6,15,26]$. However, it is important to note that neither Peschek et al. [26] nor McCormick et al. [15] successfully reduced their performance measures with their exercise protocol, thus providing no performance decrement for the supplements to attenuate. The final study that failed to show performance improvement by Levers et al. [6] saw a significant reduction in performance at only 1 of the 4 time points that were measured, again providing the supplement little opportunity to demonstrate effectiveness. Overall, it appears that when performance level is reduced by strenuous exercise, the overwhelming majority of studies do demonstrate an enhanced recovery of performance with polyphenol supplementation over placebo.

Even when reductions in muscle damage and soreness are observed in conjunction with enhanced recovery of physical performance measures with the use of a nutritional supplement, an individual would still need consider the benefits of the supplement over the consumption of the additional energy and whether that would fit with their intended dietary intake. On this note, the overwhelming majority of the literature pertaining to the effects of cherry derived antioxidants on muscle damage and performance have used fruit juices, containing at least $100 \mathrm{kcal}$ of energy, with all using multiple doses (2-3 per day) over many days (up to 10 days) $[3,11-16]$. Very few studies have assessed the effects of the antioxidant containing extracts in the absence of any macronutrients, although they have suggested potential benefits $[6,28]$. The use of tart cherry supplementation in the absence of added macronutrients has involved consuming the supplement in powder form $[6,28]$, as opposed to juice form containing significant amounts of sugar. Interestingly, these powder studies used considerably lower doses of polyphenols (500 mg vs. > $1000 \mathrm{mg}$ ) but still demonstrated ergogenic effects [6, 28].

The primary purpose of this study, using a randomized crossover design, was to assess whether polyphenol supplementation in the form of a powdered tart cherry extract reduces oxidative stress, skeletal and cardiac muscle damage, and muscle soreness following intense bilateral resistance exercise. The secondary purpose was to determine whether the recovery of physical performance was enhanced as a result. We hypothesized the polyphenol supplementation would result in reduced muscle damage and soreness and the recovery would be enhanced following intense resistance exercise.

\section{Methods}

\section{Subject Background and Preparation}

Thirteen men (mean age: $26.2 \pm 5.3$ years; height: $184.3 \pm 8.2 \mathrm{~cm}$; weight: $92.9 \pm 15.6 \mathrm{~kg}$; barbell back squat 1RM: $146.8 \pm 30.6 \mathrm{~kg}$ ) with a minimum of 6 months of prior experience in the barbell back squat completed the study. All subjects provided written informed consent after approval for the study was granted by the Institutional Review Board at Jacksonville University.

\section{Experimental Overview}

The study utilized a randomized, cross-over, counterbalanced, placebo controlled design, as illustrated in Fig. 1. Subjects initially attended familiarization and preliminary testing. During this visit, subjects were introduced to the facility and walked through the basic procedures, including muscle soreness assessment, the warm-up protocol, and the vertical jump and handgrip dynamometer procedures, followed by a 1-repetition maximum assessment on the barbell back squat.

For muscle soreness, subjects were asked to report the amount of muscle soreness that they were currently perceiving in their upper legs. While the subject was seated with legs elevated, a researcher palpated with both hands the upper, middle and lower quadriceps, applying firm pressure with the thumb and forefinger for approximately $1 \mathrm{~s}$. The subject then marked a line on a $10 \mathrm{~cm}$ scale that corresponded to their muscular soreness. The warm-up consisted of $5 \mathrm{~min}$ on a Monark exercise bike (Vansbro, Sweden) keeping a constant speed of 60-80 rpm. Following the exercise bike, subjects performed a range of dynamic stretching exercises, including bodyweight squats, lunges, lateral lunges, knee hugs, quad pulls and straight leg marches. Each dynamic stretch was performed for 10 repetitions.

Vertical jump was used to assess the impact of the muscle damage protocol on muscle performance and to identify if the supplement could enhance recovery of this measure. For the vertical jump assessment, subjects were instructed to stand on a NeuroCom Balance Manager forceplate (Natus, Pleasanton, CA) with hands on hips. Subjects were instructed to perform 3 consecutive vertical jumps, as high as possible with minimal time between jumps. Jumps were later analyzed for power using the equation described by Sayers et al. [29].

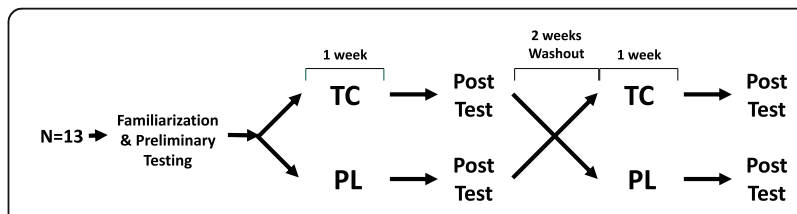

Fig. 1 Study Design. TC Tart Cherry Supplementation, PL Placebo 
As grip strength has been previously demonstrated to be associated with central fatigue [30, 31], it was used as a marker of recovery. Grip strength of the dominant hand was measured with a grip strength dynamometer (Hydraulic Hand Dynamometer, North Coast Medical Inc., Morgan Hill, USA). The highest score of three attempts was used in the analyses.

For the 1-Repetition Maximum Testing (1RM), subjects performed squats for $8-10$ repetitions at $\sim 50 \%$ of estimated 1RM followed by another set of 2-5 repetitions at $\sim 85 \%$ of 1 RM. Subsequently, 3-5 maximal trials were used to determine the individual's $1 \mathrm{RM}$, each separated by 3 min of rest. Necessary squat depth was standardized as the upper thigh reaching at minimum a position that was parallel to the ground. The highest load lifted for 1 repetition with correct technique was considered their 1RM.

\section{Supplementation}

Following the familiarization visit, subjects took the supplement daily for 1 week prior to the acute resistance exercise protocol (AREP). A large body of evidence has shown that supplementing with polyphenols daily for 3 or more days prior to and following exercise will enhance recovery [9]. The experimental supplement was a specialized, proprietary broad spectrum Tart Cherry Extract Powder (NordicCherry ${ }^{\circledR}$ ) manufactured by Specnova, LLC. The supplement contained a broad spectrum of polyphenols with total polyphenols $5-6 \%$ w/w tested via F-C assay. The subjects received a dose of $500 \mathrm{mg}$ in capsule form containing $1.3 \mathrm{kcal}, 0.3 \mathrm{~g}$ carbohydrate, $0 \mathrm{~g}$ fat and $0.008 \mathrm{~g}$ protein. For the placebo, the subjects received rice flour. Both the experimental and placebo supplements were contained in opaque capsules, size 00, thus blinding the subjects to their supplement. Participants were provided with the exact number of pills required to be taken from the time they received the supplement until their next visit to the laboratory, and asked to return the empty container. No side effects related to the supplement of any kind were reported during the study. The supplement and placebo were prepared by Specnova, LLC.

\section{Acute Resistance Exercise Protocol (AREP)}

Subjects arrived at the Exercise Physiology Laboratory following an overnight fast. Subjects were encouraged to drink 2 cups of water the evening prior and 2 cups of water the morning of the visit to ensure adequate hydration. Before, immediately following, $1 \mathrm{~h}$ and $3 \mathrm{~h}$ after the protocol, blood was drawn from an antecubital vein into a serum vacutainer.

Once the baseline blood sample was taken, the subject began the warm up, as described earlier. Following the warm up, the barbell was loaded with a weight corresponding to $80 \%$ of their predetermined 1-repetition maximum. The subject then aimed to perform 10 repetitions of the barbell back squat for 6 sets interspersed with $2 \mathrm{~min}$ of rest. If the subject was unable to complete 10 repetitions for a given set, the subsequent set was then performed with 75\% 1RM. This procedure of reducing the load 5\% 1RM for each set where the subject fails to complete 10 repetitions was repeated for all sets. When the AREP was completed on the second occasion, the exact protocol that the subject followed (in terms of weight lifted and repetition number for each set) during the first AREP was repeated. Following completion of the AREP, an immediate post-blood was taken. Subsequent blood draws also took place $1 \mathrm{~h}$ and $3 \mathrm{~h}$ following the protocol.

\section{Recovery Visits}

At 24 and $48 \mathrm{~h}$ after the AREP, a single blood draw was conducted followed by a measurement of handgrip strength and jump power. The blood draw took place following a 10-min seated rest period. During the 10min rest period, muscular soreness was assessed with a $10 \mathrm{~cm}$ visual analog scale. Handgrip strength and vertical jump power were then assessed with the procedures previously described.

\section{Week Washout}

Following the first cycle of testing, the subjects underwent a 2 week washout period. During this time no supplement was consumed and the subjects were instructed to resume their normal diet. Following the two week washout, the subjects completed the entire data collection process a second time (7 day loading period, AREP and recovery visits) with the alternate condition (i.e., supplement or placebo).

\section{Serum Analyses}

The initial blood draw prior to the AREP took place following $10 \mathrm{~min}$ of seated rest to ensure a true resting blood draw. Other than at the Immediate Post (IP) time point, all draws were conducted following a minimum of $10 \mathrm{~min}$ of seated rest. Blood was spun at $1500 \times \mathrm{g}$ for $10 \mathrm{~min}$ at room temperature and serum was aliquoted and stored at $-80{ }^{\circ} \mathrm{C}$ until subsequent analyses. Serum was analyzed in bulk at the completion of the study in our laboratory. Protein carbonyls (PC) were quantified by an Enzyme-Linked Immunosorbent Assay (ELISA) kit (OxiSelect ${ }^{\mathrm{TM}}$ Protein Carbonyl ELISA Kit, Cell Biolabs, Inc., San Diego, CA). Serum protein concentration was determined for each sample using the Bradford Protein Assay (Bio Basic, Amherst, NY) prior to running the ELISA and samples were run in triplicate per manufacturer's protocol.

Creatine kinase (CK) activity was assessed using a colorimetric assay (Creatine Kinase Activity Assay Kit, Abcam, Cambridge, MA). Reference values for CK are 
38-174 U.L $\mathrm{L}^{-1}$. Serum samples were diluted 1:5 and readings were taken over time for $10 \mathrm{~min}$. Sample preparation and analysis were performed per manufacturer's protocol.

Creatine kinase isoenzyme MB was assayed by ELISA per manufacturer's protocol (Human CKMB (CKM/ CKB) ELISA Kit, Thermo Scientific, Frederick, MD). Reference values for CKMB are $0-10 \mathrm{ng} \cdot \mathrm{ml}^{-1}$.

\section{Statistical Analyses}

All data are presented as means along with standard deviation. Data were confirmed to demonstrate a normal distribution according to Shapiro-Wilk. Data points greater than 2 standard deviations from the mean were removed as outliers. Less than $2.5 \%$ of the data were removed as outliers. If the baseline value for a subject was not attainable for a certain variable, the subject was removed from analysis for that variable. Missing data was replaced with the mean delta for that time point. A 2-way repeated measures ANOVA was conducted for PC, CK and CKMB. A 2way repeated measures ANOVA was conducted for muscle soreness, jump power and handgrip dynamometer measurements. The Mauchley sphericity test was conducted to confirm homogeneity of variance; if this assumption was violated, the Greenhouse-Geisser adjustment was performed If statistical significance was observed, post-hoc analyses were conducted in the form of paired $t$-tests on the change from baseline values at each time point, with a Bonferroni correction factor to account for alpha inflation. All statistics were run using SPSS Statistical Software, Version 24 (IBM Corporation, Armonk, NY, USA).

\section{Results}

Raw measures of oxidative stress, muscle damage and physical performance parameters are illustrated in Table 1.

\section{Oxidative Stress}

There was a significant main effect for supplement $(p=0.019)$, but not time $(p=0.520)$ and also a significant supplement"time interaction $(p<0.001)$. Figure 2 illustrates the changes in PC at each time point compared to baseline. There was a statistically significant increase in $\mathrm{PC}$ in the Placebo Condition when compared to the Tart Cherry Condition at IP (PL: 0.4 \pm 0.3 vs. TC: $-0.4 \pm 0.2 \mathrm{nmol} \cdot \mathrm{mg}^{-1} ; p<0.001$ ), $1 \mathrm{~h}$ (PL: $0.3 \pm 0.3$ vs. TC: $\left.-0.7 \pm 0.3 \mathrm{nmol} \cdot \mathrm{mg}^{-1} ; p<0.001\right)$ and 24. (PL: $0.1 \pm 0.4$ vs. TC: $-0.3 \pm 0.5 \mathrm{nmol} \cdot \mathrm{mg}^{-1} ; p=$ $0.010)$.

\section{Muscle Damage \\ Creatine Kinase (CK) Activity}

There was a significant main effect for supplement ( $p=$ $0.036)$, time $(p<0.001)$ and also a significant supplement"time interaction $(p=0.003)$. Figure 3 illustrates the changes in $\mathrm{CK}$ activity at each time point compared to baseline. There was a statistically significant increase in CK activity in the Placebo Condition when compared to the Tart Cherry Condition at IP (PL: $491.1 \pm 280$ vs. TC: $\left.296.3 \pm 178 \mathrm{U} \cdot \mathrm{L}^{-1} ; p=0.008\right)$ and $3 \mathrm{~h}$ (PL: $-87 \pm 123$ vs. TC: $\left.43.1 \pm 105.3 \mathrm{U} \cdot \mathrm{L}^{-1} ; p=0.006\right)$.

\section{Creatine Kinase Myocardial Band (CK-MB)}

There was a significant main effect for time $(p<0.001)$, and also a significant supplement"time interaction $(p=$ $0.001)$, but no main effect for supplement $(p=0141)$.

Table 1 Raw measures of oxidative stress, muscle damage and physical performance parameters for 13 men following a heavy resistance exercise protocol after supplementing with placebo and tart cherry supplementation

\begin{tabular}{|c|c|c|c|c|c|c|c|}
\hline & \multirow[b]{2}{*}{ Group } & \multicolumn{6}{|l|}{ Time Point } \\
\hline & & $\mathrm{BL}$ & IP & $1 \mathrm{~h}$ & $3 \mathrm{~h}$ & $24 \mathrm{~h}$ & $48 \mathrm{~h}$ \\
\hline \multirow[t]{2}{*}{$\mathrm{PC}\left(\mathrm{nmol} \cdot \mathrm{mg}^{-1}\right)$} & $\mathrm{TC}$ & $2.43(0.73)$ & $2.01(0.65)$ & $1.73(0.76)$ & $2.14(0.88)$ & $2.09(0.58)$ & $2.23(0.66)$ \\
\hline & $P$ & $2.03(0.52)$ & $2.5(0.7)$ & $2.44(0.57)$ & $2.34(0.64)$ & $2.23(0.56)$ & $2.37(0.67)$ \\
\hline \multirow[t]{2}{*}{ CK $\left(\left.U \cdot\right|^{-1}\right)$} & $\mathrm{TC}$ & $254(106)$ & $602(253)$ & $320(126)$ & $297(110)$ & $299(89)$ & $235(95)$ \\
\hline & $P$ & $322(131)$ & $813(256)$ & $343(148)$ & 269 (138) & $354(111)$ & 305 (139) \\
\hline \multirow[t]{2}{*}{ CKMB $\left(\mathrm{ng} \cdot \mathrm{ml}^{-1}\right)$} & $\mathrm{TC}$ & $22(11)$ & $20(5)$ & $21(7)$ & $20(6)$ & $23(11)$ & $20(10)$ \\
\hline & $P$ & $21(9)$ & $24(11)$ & $43(18)$ & $27(10)$ & $20(7)$ & $13(9)$ \\
\hline \multirow[t]{2}{*}{ Soreness (cm) } & $\mathrm{TC}$ & $0.6(0.6)$ & & & & $4.5(2.2)$ & $3.9(1.8)$ \\
\hline & $P$ & $0.7(0.7)$ & & & & $5.7(1.9)$ & $5.9(2.7)$ \\
\hline \multirow[t]{2}{*}{ Jump Power (W) } & $\mathrm{TC}$ & 3921 (869) & & & & 3699 (946) & 3857 (887) \\
\hline & $P$ & 3841 (878) & & & & 3897 (763) & 3856 (803) \\
\hline \multirow[t]{2}{*}{ Handgrip (Kg) } & $\mathrm{TC}$ & $47(6)$ & & & & $49(6)$ & $49(6)$ \\
\hline & $P$ & $50(5)$ & & & & $48(6)$ & $50(5)$ \\
\hline
\end{tabular}

TC Tart Cherry, P Placebo, PC Protein Carbonyls, CK Creatine Kinase, BL Baseline, IP Immediately Post, $1 \mathrm{~h}=1 \mathrm{~h}$ post; $3 \mathrm{~h}=3 \mathrm{~h}$ post; $24 \mathrm{~h}=24 \mathrm{~h}$ post; $48 \mathrm{~h}=48 \mathrm{~h}$ post 


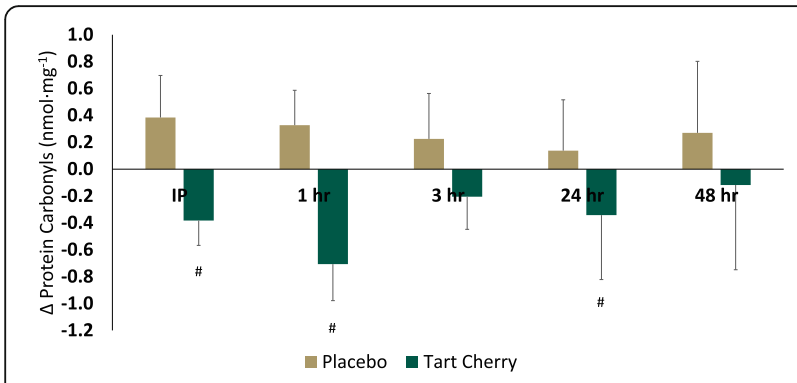

Fig. 2 Changes in Protein Carbonyls (PC) from baseline for 13 men following a heavy resistance exercise protocol after supplementing with placebo and tart cherry supplementation. \# = significantly $(p \leq$ 0.05) different from placebo at corresponding time point. IP Immediately Post; $1 \mathrm{~h}=1 \mathrm{~h}$ post; $3 \mathrm{~h}=3 \mathrm{~h}$ post; $24=24 \mathrm{~h}$ post; $48=48$ h post

Figure 4 illustrates the changes in CKMB at each time point compared to baseline. There was a statistically significant $(p=0.003)$ increase in CKMB concentration in the Placebo Group when compared to the Tart Cherry group (PL: $21.6 \pm 12.4$ vs. TC: $-0.3 \pm 11.8 \mathrm{ng} \cdot \mathrm{ml}^{-1}$; $\mathrm{p}=$ $0.006)$ at $1 \mathrm{~h}$ post. There were no other statistically significant differences.

\section{Muscle Soreness}

There was a significant main effect for time $(p<0.001)$, but a non-significant main effect for supplement $(p=$ $0.136)$ and supplement"time interaction $(p=0.158)$. Muscle soreness changes at each time point when compared to baseline are displayed in Fig. 5. There were no other statistically significant differences.

\section{Physical Performance}

There was a non-significant main effect for time ( $p=$ $0.396)$ and main effect for supplement $(p=0.145)$, but a significant supplement*time interaction $(p=0.041)$ for handgrip strength. Also, there was a non-significant main effect for time $(p=0.381)$ and main effect for supplement $(p=0.688)$, but a significant supplement*time

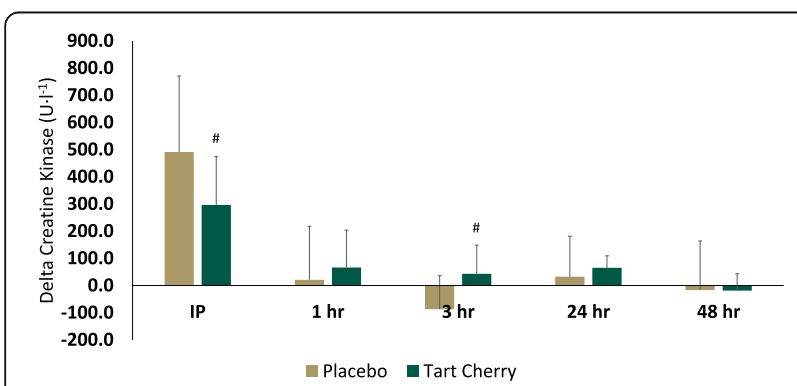

Fig. 3 Changes in Creatine Kinase (CK) activity from baseline for 13 men following a heavy resistance exercise protocol after supplementing with placebo and tart cherry supplementation. \# = significantly $(p \leq 0.05)$ different from placebo at corresponding time point. IP Immediately Post; 1 $\mathrm{h}=1 \mathrm{~h}$ post; $3 \mathrm{~h}=3 \mathrm{~h}$ post; $24=24 \mathrm{~h}$ post $; 8=48 \mathrm{~h}$ post

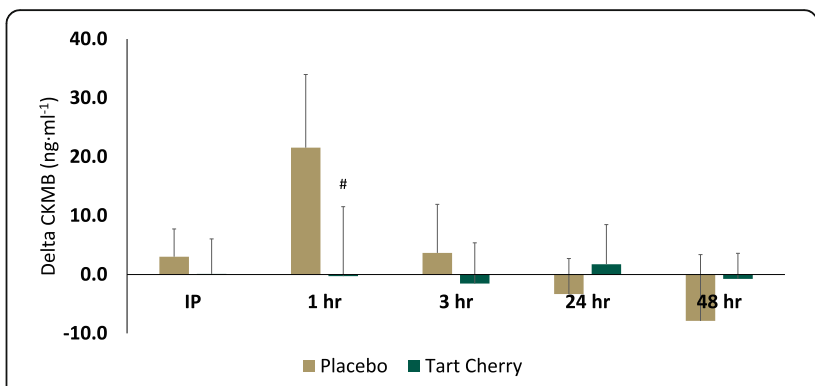

Fig. 4 Changes in Creatine Kinase-MB (CKMB) concentrations from baseline for 13 men following a heavy resistance exercise protocol after supplementing with placebo and tart cherry supplementation. \# = significantly $(p \leq 0.05)$ different from placebo at corresponding time point. IP $=$ Immediately Post; $1 \mathrm{~h}=1 \mathrm{~h}$ post; $3 \mathrm{~h}=3 \mathrm{~h}$ post; $24=$ $24 \mathrm{~h}$ post; $48=48 \mathrm{~h}$ post

interaction $(p=0.020)$ for jump power. Table 2 illustrates the changes in physical performance parameters at each time point when compared to baseline. There was a statistically significant increase in handgrip strength in the Tart Cherry group when compared to the Placebo Group (PL: $-2 \pm 5.1$ vs. TC: $1.7 \pm 3 \mathrm{~kg} ; p=0.017$ ) at $24 \mathrm{~h}$ post. There were no statistically significant differences, although jump height was trending $(p=0.073)$ towards significance (PL: $-7.3 \pm 112.9$ vs. TC: $-207.7 \pm 291.5 \mathrm{~W})$.

\section{Discussion}

The primary finding from this study is that the specialized, proprietary tart cherry supplementation was able to reduce oxidative stress and subsequently both skeletal and cardiac muscle damage, as well as reduce the attenuation of grip strength following an intense resistance exercise protocol when compared to placebo. This was achieved with extract containing minimal energy intake (1.3 kcal).

Paradoxically, while exercise is a powerful stimulus that can lead to positive changes in health and performance, it does induce an oxidative stress [32]. The typical

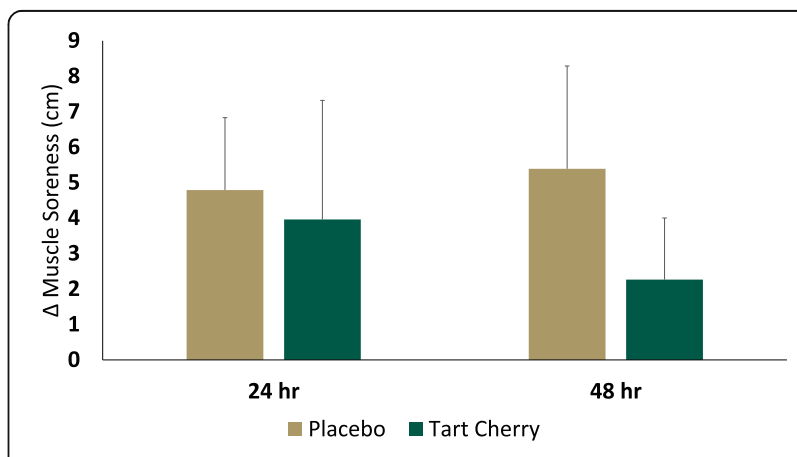

Fig. 5 Changes in muscle soreness values from baseline for 13 men following a heavy resistance exercise protocol after supplementing with placebo and tart cherry supplementation. $24 \mathrm{~h}=24 \mathrm{~h}$ post; $48 \mathrm{~h}=48 \mathrm{~h}$ post 
Table 2 Physical Performance. Changes in physical performance parameters for 13 men following a heavy resistance exercise protocol after supplementing with placebo and tart cherry supplementation

\begin{tabular}{|c|c|c|c|c|c|c|}
\hline \multirow[b]{2}{*}{ Variable } & \multirow{2}{*}{$\begin{array}{l}\text { Group } \\
\text { Time Point }\end{array}$} & \multicolumn{2}{|l|}{ PL } & \multicolumn{3}{|l|}{$\mathrm{TC}$} \\
\hline & & Mean & SD & Mean & SD & $p$ \\
\hline \multirow[t]{2}{*}{ Jump Power (W) } & $\Delta 24 \mathrm{~h}$ & -7.3 & 112.9 & -207.7 & 291.5 & 0.073 \\
\hline & $\triangle 48 \mathrm{~h}$ & 66.7 & 204.6 & -59.8 & 329.8 & 0.692 \\
\hline \multirow[t]{2}{*}{ Handgrip Dynamometer (Kg) } & $\Delta 24 \mathrm{~h}$ & -2.0 & 5.1 & $1.7 \#$ & 3.0 & 0.034 \\
\hline & $\triangle 48 \mathrm{~h}$ & 0.5 & 3.7 & 2.5 & 3.3 & 0.627 \\
\hline
\end{tabular}

\# = significantly $(P \leq 0.05)$ different from placebo at corresponding time point. $24 \mathrm{~h}=24 \mathrm{~h}$ post; $48 \mathrm{~h}=48 \mathrm{~h}$ post

increase in oxidative stress seen following intense exercise that was demonstrated in the placebo group was completely nullified by the supplement, as seen by negative PC delta scores at each time point (Fig. 2. These changes in PC have been demonstrated previously, both following resistance [3] and aerobic [17] exercise utilizing Montmorency cherry and purple sweet potato supplementation respectively. While Levers et al. [6] did not demonstrate changes in oxidative stress following tart cherry supplementation despite showing significant changes in muscle damage, the investigators did not address PC levels in that study. Thus, our data proposes PC may be a more sensitive indicator of changes in oxidative stress following polyphenol supplementation.

While an antioxidant supplement is unable to affect the mechanical stress placed on muscle tissue, by scavenging reactive oxygen species the supplement was able to reduce the amount of biochemical damage placed on the muscle proteins, as evidenced by significant decreases in both CK activity and CKMB. Although other polyphenol containing supplements such as blackcurrant juice [23] have demonstrated a reduction in skeletal muscle damage, this has not been previously shown in the tart cherry supplementation studies. In addition to skeletal muscle damage, this study assessed a marker of cardiac muscle damage [5], CKMB which was recently identified as a potential marker of type I muscle fiber damage [18]. While CKMB has been shown to decrease in the presence of other antioxidants, such as vitamin $\mathrm{E}$ [20] and Allopurinol [19], this study provides evidence that the compounds and fractions in tart cherries, including polyphenols, may have the same effect. CKMB may also be more sensitive to changes in muscle damage than CK activity following antioxidant supplementation, as several studies have failed to show differences in muscle damage following marathon running [16], cycling [12], intermittent sprints [10], or unilateral knee extension [3]. All of these prior studies utilized CK activity alone as their measure of muscle damage, rather than both CK activity and CKMB together, as we have done here. It is also noteworthy that although the supplement attenuated the increase in CK following the AREP, the increase CK was generally mild and would be categorized as low by prior studies [33, 34]. A more demanding AREP could have led to greater and more prolonged increases in CK, which could have given more opportunities for the supplement to demonstrate its effects.

Prior research has previously demonstrated reductions in muscle soreness $[10,13,14]$ associated with antioxidant supplementation, however, these changes were not seen in this study (Fig. 5). Despite the reduction in oxidative stress and subsequent reduction in muscle damage, these were not manifested as perceivable changes in soreness to the subjects in this experiment. Other studies have also failed to demonstrate these changes in soreness even in the presence of attenuation in inflammation and oxidative damage [16], which ultimately illustrates the subjectivity and difficulty in quantifying soreness [9].

With regard to muscle damage, there may be a delicate balance. Muscle damage is a typical response to intense resistance exercise, following which the body recovers and adapts. However, if the recovery is inadequate, this can lead to a reduction in performance in the form of non-functional overreaching or overtraining [7]. Although this acute study was too short to induce non-functional overreaching, there was a significant attenuation of loss grip strength (Table 2). Loss of grip strength has previously been associated with central fatigue [30,31], where reductions in grip strength were not seen from changes within the muscle, but rather from changes within the brain [31]. Thus, although speculative, it is possible that the fatigue induced by the AREP led to a reduction in the strength of the signal to produce force in the grip strength task in placebo group, but due to less oxidative stress and muscle damage in the TC group, this response did not occur and grip strength was maintained.

It is somewhat surprising that the supplement could have an effect on grip strength and not jump power considering the nature of the exercise protocol was the squat exercise. However, despite the intense lower body exercise, jump performance was not attenuated during the recovery visits, giving the supplement no performance decrements on which to improve. In a similar 
study, Levers et al. [6] utilized 70\% 1RM in the barbell back squat as the muscle damage protocol, but did successfully induce reductions in performance in the form of maximal voluntary contractions $24 \mathrm{~h}$ following. Levers et al. [6], utilized 10 sets of squats, whereas this study used only 6 sets by comparison. This reduced volume is likely the reason for the failure for this study to induce performance decrements, particularly as the subjects in the two studies had remarkably similar strength levels (this study: $146.8 \pm 30.6$ vs. $142.2 \pm 32.2 \mathrm{~kg}$ ).

With regards to the limitations of this study, the exact amount of antioxidants consumed by the subjects is not known as there was while the subjects were encouraged to repeat their dietary intake during both arms of the study, this was not prescribed or monitored. In addition, the subjects were provided with the supplement and instructed to consume it, but they were not directly supervised ingesting the supplement. In terms of assessing oxidative stress, there are many ways this can be done, however this study only utilized one method in protein carbonyls. Other measures of oxidative stress could certainly have demonstrated different results. Finally, it appears the muscle damage protocol used in this study was mild in terms of either its intensity or volume as it produced only a modest increase in CK and failed to attenuate CMJ performance. In addition, the CK values reported may have been confounded by acute changes in plasma volume, which have been previously reported to occur with resistance training $[35,36]$. If plasma volume changes had been measured in this study, the values could have been corrected accordingly.

\section{Conclusions}

Polyphenol supplementation in the form of tart cherries has been shown to reduce oxidative stress and markers of muscle damage following intense resistance exercise. In addition, much like other plant compound supplements, polyphenols were shown to reduce CKMB, a marker of cardiac muscle damage. These declines in oxidative stress and muscle damage were associated with a significant strength benefit by preventing the decrease in strength seen following the intense exercise protocol, indicating a potential reduction in central fatigue. These benefits were seen with minimal energy intake. However, the protocol was not sufficient to cause reductions in power performance, and thus the supplement was unable to demonstrate reduced attenuations of performance as a result of the decreased damage. In the future, chronic studies are needed to show whether these reduced muscle damage markers and improved recovery of strength will translate to increases in long-term performance, or whether reducing the muscle damage markers could alter the necessary damage and recovery cycle needed for optimal adaptation.

\begin{abstract}
Abbreviations

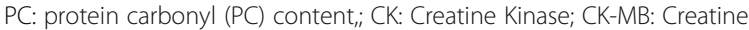
Kinase Myocardial Band; TC: Tart Cherry; PL: Placebo; 1RM: 1-Repetition Maximum; AREP: Acute Resistance Exercise Protocol; ELISA: Enzyme-Linked Immunosorbent Assay kit
\end{abstract}

\section{Acknowledgements}

The authors would like to acknowledge the substantial commitment that was required from the subjects. The authors would also like to thank Scott Powers and Joanna Bowtell for their consultation in study design.

\section{Authors' Contributions}

$\mathrm{DRH}$ designed the study, wrote the grant, collected and analyzed data and wrote the manuscript. TO collected and analyzed data. MG, AD and KC collected data and performed all biochemical analyses. $\mathrm{KC}$ and $\mathrm{HH}$ wrote substantial sections of the manuscript and contributed to study design.

\section{Funding}

This study was funded by Specnova, Inc. (Gainesville, FL, USA).

\section{Availability of Data and Materials}

The datasets used and/or analysed during the current study are available from the corresponding author on reasonable request.

\section{Declarations}

Ethics Approval and Consent to Participate

All subjects gave written informed consent and this study was approved by Jacksonville University Institutional Review Board.

Consent for Publication

Not applicable.

\section{Competing Interests}

This study was funded by Specnova, Inc. (Gainesville, FL, USA). All researchers involved independently collected, analyzed, and interpreted the results from this study and have no financial interests concerning the outcome of this investigation. The results from this study do not constitute endorsement by the authors and/or the institution concerning the nutrients investigated.

\section{Author details}

${ }^{1}$ Center for Health and Human Performance, Jacksonville University, Jacksonville, USA. ²Department of Kinesiology, Brooks Rehabilitation College of Healthcare Sciences, Health Sciences Complex, Jacksonville University, 2800 University Boulevard North, Jacksonville, FL 32211, USA. ${ }^{3}$ Department of Biology and Marine Science, Jacksonville University, Jacksonville, USA.

Received: 9 August 2020 Accepted: 2 June 2021

Published online: 14 June 2021

\section{References}

1. American College of Sports Medicine Position Stand. Progression models in resistance training for healthy adults. Med Sci Sports Exerc. 2009;41(3):687-708.

2. Hudson MB, Hosick PA, McCaulley GO, Schrieber L, Wrieden J, McAnulty SR, et al. The effect of resistance exercise on humoral markers of oxidative stress. Med Sci Sports Exerc. 2008;40(3):542-8. https://doi.org/10.1249/MSS. 0b013e31815daf89.

3. Bowtell $\lrcorner$, Sumners DP, Dyer A, Fox P, Mileva KN. Montmorency cherry juice reduces muscle damage caused by intensive strength exercise. Med Sci Sports Exerc. 2011;43(8):1544-51. https://doi.org/10.1249/MSS.0b013e31 $820 \mathrm{e} 5 \mathrm{adc}$.

4. Heavens KR, Szivak TK, Hooper DR, Dunn-Lewis C, Comstock BA, Flanagan $\mathrm{SD}$, et al. The effects of high intensity short rest resistance exercise on muscle damage markers in men and women. J Strength Cond Res. 2014; 28(4):1041-9. https://doi.org/10.1097/JSC.0000000000000236.

5. Hayward R, Hutcheson KA, Schneider CM. Influence of acute resistance exercise on cardiac biomarkers in untrained women. J Emerg Med. 2003; 25(4):351-6. https://doi.org/10.1016/S0736-4679(03)00239-7.

6. Levers K, Dalton R, Galvan E, Goodenough C, O'Connor A, Simbo S, et al. Effects of powdered Montmorency tart cherry supplementation on an acute 
bout of intense lower body strength exercise in resistance trained males. J Int Soc Sports Nutr. 2015;12(1):41. https:/doi.org/10.1186/s12970-015-0102-y.

7. Meeusen R, Duclos M, Foster C, Fry A, Gleeson M, Nieman D, et al. Prevention, diagnosis, and treatment of the overtraining syndrome: joint consensus statement of the European college of sport science and the American college of sports medicine. Med Sci Sports Exerc. 2013;45(1):186205. https://doi.org/10.1249/MSS.0b013e318279a10a.

8. Honda S, Kawasaki T, Kamitani T, Kiyota K. Rhabdomyolysis after high intensity resistance training. Intern Med. 2017;56(10):1175-8. https://doi. org/10.2169/internalmedicine.56.7636.

9. Bowtell J, Kelly V. Fruit-derived polyphenol supplementation for athlete recovery and performance. Sports Med. 2019;49(Suppl 1):3-23. https://doi. org/10.1007/s40279-018-0998-x.

10. Bell PG, Stevenson E, Davison GW, Howatson G. The effects of montmorency tart cherry concentrate supplementation on recovery following prolonged, intermittent exercise. Nutrients. 2016;8(7):441.

11. Bell PG, Walshe IH, Davison GW, Stevenson E, Howatson G. Montmorency cherries reduce the oxidative stress and inflammatory responses to repeated days high-intensity stochastic cycling. Nutrients. 2014;6(2):829-43. https://doi.org/10.3390/nu6020829.

12. Bell PG, Walshe IH, Davison GW, Stevenson EJ, Howatson G. Recovery facilitation with Montmorency cherries following high-intensity, metabolically challenging exercise. Appl Physiol Nutr Metab. 2015;40(4):41423. https://doi.org/10.1139/apnm-2014-0244

13. Connolly DA, McHugh MP, Padilla-Zakour OI, Carlson L, Sayers SP. Efficacy of a tart cherry juice blend in preventing the symptoms of muscle damage. $\mathrm{Br}$ J Sports Med. 2006;40(8):679-83; discussion 83. https://doi.org/10.1136/ bjsm.2005.025429.

14. Kuehl KS, Perrier ET, Elliot DL, Chesnutt JC. Efficacy of tart cherry juice in reducing muscle pain during running: a randomized controlled trial. J Int Soc Sports Nutr. 2010;7(1):17. https://doi.org/10.1186/1550-2783-7-17.

15. McCormick R, Peeling P, Binnie M, Dawson B, Sim M. Effect of tart cherry juice on recovery and next day performance in well-trained Water Polo players. J Int Soc Sports Nutr. 2016;13(1):41. https://doi.org/10.1186/s12970016-0151-x

16. Howatson G, McHugh MP, Hill JA, Brouner J, Jewell AP, van Someren KA, et al. Influence of tart cherry juice on indices of recovery following marathon running. Scand J Med Sci Sports. 2010;20(6):843-52. https://doi. org/10.1111/j.1600-0838.2009.01005.x.

17. Chang WH, Hu SP, Huang YF, Yeh TS, Liu JF. Effect of purple sweet potato leaves consumption on exercise-induced oxidative stress and IL-6 and HSP72 levels. J Appl Physiol (1985). 2010;109(6):1710-5.

18. Carmona G, Roca E, Guerrero M, Cusso R, Barcena C, Mateu M, et al. Fibretype-specific and mitochondrial biomarkers of muscle damage after mountain races. Int J Sports Med. 2019;40(4):253-62. https://doi.org/10.1 055/a-0808-4692.

19. Sanchis-Gomar F, Pareja-Galeano H, Gomez-Cabrera MC, Candel J, Lippi G, Salvagno GL, et al. Allopurinol prevents cardiac and skeletal muscle damage in professional soccer players. Scand J Med Sci Sports. 2015;25(1):e110-5. https://doi.org/10.1111/sms.12213.

20. Santos SA, Silva ET, Caris AV, Lira FS, Tufik S, Dos Santos RV. Vitamin E supplementation inhibits muscle damage and inflammation after moderate exercise in hypoxia. J Hum Nutr Diet. 2016;29(4):516-22. https://doi.org/1 $0.1111 /$ jhn. 12361.

21. Ives SJ, Bloom S, Matias A, Morrow N, Martins N, Roh Y, et al. Effects of a combined protein and antioxidant supplement on recovery of muscle function and soreness following eccentric exercise. J Int Soc Sports Nutr. 2017;14(1):21. https://doi.org/10.1186/s12970-017-0179-6.

22. Romain C, Freitas TT, Martinez-Noguera FJ, Laurent C, Gaillet S, Chung LH, et al. Supplementation with a polyphenol-rich extract, tensless ${ }^{\oplus}$, attenuates delayed onset muscle soreness and improves muscle recovery from damages after eccentric exercise. Phytother Res. 2017;31(11):1739-46. https://doi.org/10.1002/ptr.5902.

23. Hutchison AT, Flieller EB, Dillon KJ, Leverett BD. Black currant nectar reduces muscle damage and inflammation following a bout of high-intensity eccentric contractions. J Diet Suppl. 2016;13(1):1-15. https://doi.org/10.31 09/19390211.2014.952864.

24. Trombold JR, Barnes JN, Critchley L, Coyle EF. Ellagitannin consumption improves strength recovery 2-3 d after eccentric exercise. Med Sci Sports Exerc. 2010:42(3):493-8. https://doi.org/10.1249/MSS.0b013e3181b64edd.
25. McLeay Y, Barnes MJ, Mundel T, Hurst SM, Hurst RD, Stannard SR. Effect of New Zealand blueberry consumption on recovery from eccentric exerciseinduced muscle damage. J Int Soc Sports Nutr. 2012;9(1):19. https://doi. org/10.1186/1550-2783-9-19.

26. Peschek K, Pritchett R, Bergman E, Pritchett K. The effects of acute post exercise consumption of two cocoa-based beverages with varying flavanol content on indices of muscle recovery following downhill treadmill running Nutrients. 2013;6(1):50-62. https://doi.org/10.3390/nu6010050.

27. Trombold JR, Reinfeld AS, Casler JR, Coyle EF. The effect of pomegranate juice supplementation on strength and soreness after eccentric exercise. J Strength Cond Res. 2011;25(7):1782-8. https://doi.org/10.1519/JSC.0b013e31 8220d992.

28. Levers K, Dalton R, Galvan E, O'Connor A, Goodenough C, Simbo S, et al. Effects of powdered Montmorency tart cherry supplementation on acute endurance exercise performance in aerobically trained individuals. J Int Soc Sports Nutr. 2016;13(1):22. https://doi.org/10.1186/s12970-016-0133-z.

29. Sayers SP, Harackiewicz DV, Harman EA, Frykman PN, Rosenstein MT. Crossvalidation of three jump power equations. Med Sci Sports Exerc. 1999;31(4): 572-7. https://doi.org/10.1097/00005768-199904000-00013.

30. Nybo L, Nielsen B. Hyperthermia and central fatigue during prolonged exercise in humans. J Appl Physiol (1985). 2001;91 (3):1055-60.

31. Rasmussen P, Dawson EA, Nybo L, van Lieshout JJ, Secher NH, Gjedde A. Capillary-oxygenation-level-dependent near-infrared spectrometry in frontal lobe of humans. J Cereb Blood Flow Metab. 2007;27(5):1082-93. https://doi. org/10.1038/sj.jcbfm.9600416.

32. Powers SK, Jackson MJ. Exercise-induced oxidative stress: cellular mechanisms and impact on muscle force production. Physiol Rev. 2008; 88(4):1243-76. https://doi.org/10.1152/physrev.00031.2007.

33. Clarkson PM. Exercise-induced muscle damage--animal and human models. Med Sci Sports Exerc. 1992;24(5):510-1.

34. Chen TC. Variability in muscle damage after eccentric exercise and the repeated bout effect. Res Q Exerc Sport. 2006;77(3):362-71. https://doi.org/1 0.1080/02701367.2006.10599370.

35. Collins MA, Hill DW, Cureton KJ, DeMello JJ. Plasma volume change during heavy-resistance weight lifting. Eur J Appl Physiol Occup Physiol. 1986;55(1): 44-8. https://doi.org/10.1007/BF00422891.

36. Ahmadizad S, El-Sayed MS. The acute effects of resistance exercise on the main determinants of blood rheology. J Sports Sci. 2005;23(3):243-9. https:// doi.org/10.1080/02640410410001730151.

\section{Publisher's Note}

Springer Nature remains neutral with regard to jurisdictional claims in published maps and institutional affiliations.

Ready to submit your research? Choose BMC and benefit from:

- fast, convenient online submission

- thorough peer review by experienced researchers in your field

- rapid publication on acceptance

- support for research data, including large and complex data types

- gold Open Access which fosters wider collaboration and increased citations

- maximum visibility for your research: over $100 \mathrm{M}$ website views per year

At BMC, research is always in progress.

Learn more biomedcentral.com/submissions 